\title{
Case report: bilateral tunneled epidural catheters to prevent unilateral analgesia for cancer- related pain
}

This article was published in the following Dove Press journal:

Local and Regional Anesthesia

20 July 2017

Number of times this article has been viewed

\author{
Raj B Padalia' \\ Corey J Reeves ${ }^{2}$ \\ Neal Shah' \\ Ankur A Patel ${ }^{3}$ \\ Devang $M$ Padalia ${ }^{4}$
}

'Pain Medicine, University of South Florida, Tampa, FL, USA; ${ }^{2}$ Physical Medicine and Rehabilitation,

University of South Florida, Tampa,

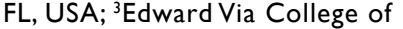
Osteopathic Medicine, Blacksburg, VA, USA; ${ }^{4}$ Interventional Pain, Moffitt Cancer Center, University of South Florida, Tampa, FL, USA
Correspondence: Raj B Padalia James A. Haley Veterans' Hospital, 13000 Bruce B. Downs Boulevard, Tampa, FL 33612, USA

Tel +18139722000 ext 7085

Fax + I 8139490116

Email rajpadalia@gmail.com
Objective: Unilateral analgesia often occurs with epidural analgesia. Traditional methods of troubleshooting this problem can be insufficient in obtaining adequate pain relief in a timely manner for terminal cancer patients. This case report demonstrates a safe and effective solution which can be utilized in these circumstances.

Case report: A 55-year-old female with stage IV pancreatic cancer and life expectancy of a few weeks presented to the interventional pain clinic with intractable sacral pain. The decision to place an epidural catheter and external pump for analgesia was made. An epidural catheter placed at the L5-S1 level showed contrast spread only along the right nerve roots and a test dose produced only right-sided analgesia. Suspecting compartmentalization of the epidural space, a second left-sided epidural catheter was placed and bilateral analgesia was achieved by using both catheters. This dual catheter technique gave the patient effective bilateral analgesia until she passed away several weeks later.

Conclusion: The bilateral epidural catheter technique is safe and effective in patients who present with persistent unilateral epidural analgesia despite exhausting traditional solutions.

Keywords: pain management, palliative care, cancer, regional techniques

\section{Introduction}

Pain is one of the most feared aspects for patients diagnosed with cancer. In the majority of cases, oral opioids and adjuvant therapy are sufficient in treating this pain. Unfortunately, a small percentage of patients do not respond to conservative management and must undergo interventional procedures to maximize their pain relief. Some techniques used in the management of cancer-related pain include neurolytic blocks as well as spinal and epidural analgesia. Spinal analgesia is the delivery of agents such as opioids and local anesthetics to the cerebrospinal fluid (CSF) usually via an implanted catheter and pump system. This is a very effective method for pain control but can be difficult to manage and titrate in an outpatient setting. Epidural analgesia via a catheter is also an effective system and more likely to be used when the life expectancy of the patient is less than 3 months, secondary to cost considerations. One particular problem is unilateral epidural analgesia from the uneven distribution of local anesthetic in the epidural space which has been described in the literature. The combination of a misplaced catheter with contributing secondary causes such as small volumes of injectate, a dorsomedian septum (plica mediana dorsalis [PMD]), or even disruption of the PMD through high pressure injection or the epidural needle may affect the restriction of epidural spread and change the likelihood of a unilateral spread of local anesthetic. ${ }^{1-4}$ Many non-uniform distributions of contrast have been 
shown to produce bilateral analgesia. ${ }^{2,3}$ What is certain is that the epidural space is not uniform throughout and it can often have significant variability which can create a significant challenge in obtaining effective epidural analgesia..$^{2-5}$ Here we present a case requiring the use of bilateral epidural catheters in order to achieve bilateral analgesia.

\section{Case report}

A 55-year-old Caucasian female with stage IV pancreatic cancer and without other significant medical history presented to the interventional pain clinic with increasing sacral, lower extremity, and pelvic pain. The patient was unable to ambulate secondary to pain and deconditioning. Magnetic resonance imaging without contrast and computed tomography (CT) with contrast just prior to her clinic visit showed a large pancreatic mass with liver infiltration, multiple pelvic and sacral metastatic lesions, and a significant collection of intraabdominal fluid. Of note, the lumbar and sacral epidural space showed no abnormalities or lesions. Her oncology team determined she was no longer a candidate for any further treatment and recommended palliative measures only. Her prognosis was poor with a life expectancy of a few weeks. Her pain control regimen included fentanyl patch $200 \mathrm{mcg}$ every 72 hours, hydrocodone/acetaminophen $10 / 325 \mathrm{mg}$ every 6 hours as needed, duloxetine $60 \mathrm{mg}$ daily, and gabapentin $300 \mathrm{mg}$ three times a day; however, she continued to have uncontrolled pain with significant decrease in her quality of life.

Due to the patient's poor prognosis and short life expectancy, an implantable intrathecal pain pump was not pursued. It was decided that a tunneled multiorifice epidural catheter with an external infusion pump would be the best treatment option for her pain. The epidural catheter was placed at the L5-S1 level using standard fluoroscopic technique. A paramedian approach with the Tuohy bevel directed cephalad was used to advance the catheter to the L3-4 vertebral level. Then $2 \mathrm{~mL}$ of injected contrast showed spread along the right L5 and S1 nerve roots. There was no contralateral spread seen (Figure 1) and therefore the decision was made to inject a test dose of $6 \mathrm{~mL}$ of bupivacaine $0.25 \%$. After waiting for 10 minutes, the patient was assessed and she had marked anesthesia and decreased pain on her right side, but normal pain on the left. To get full relief of her symptoms, it was decided to place a second tunneled epidural catheter using the same technique as the previous catheter placement along the left side of the epidural space at the same L5-S1 level in order to achieve bilateral analgesia. Then $2 \mathrm{~mL}$ of contrast was again injected through this catheter after placement, and it showed spread only along the left L5 and S1 nerve

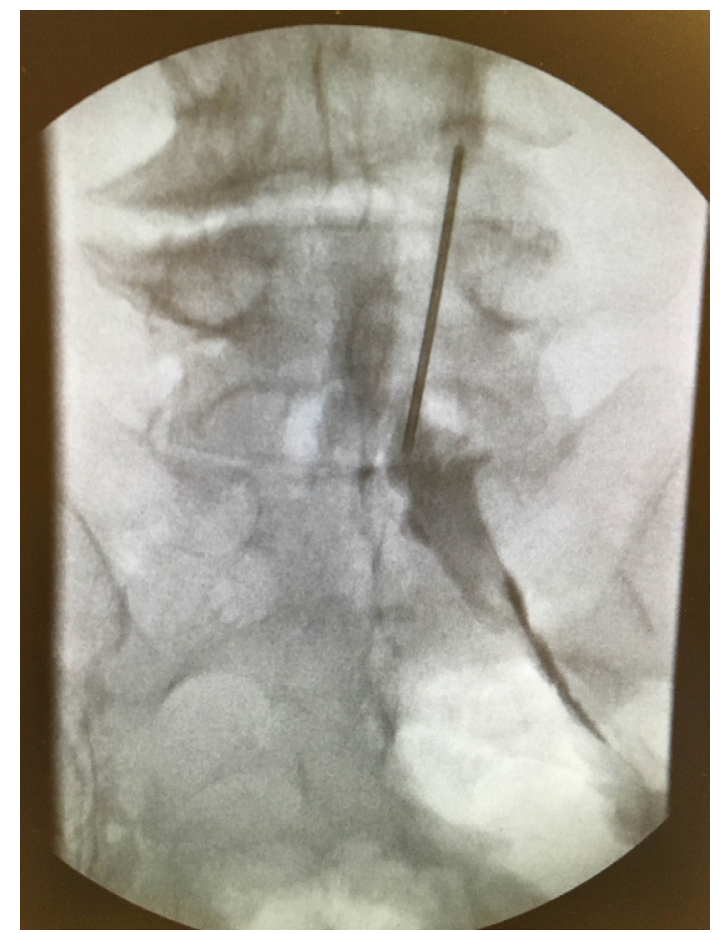

Figure I Anteroposterior fluoroscopic image of contrast injected through the first epidural catheter which was placed showing spread only to right-sided nerve roots.

roots (Figure 2). To achieve contrast spread bilaterally, injection through both epidural catheters was required. The placement of two epidural catheters was therefore performed in order to avoid the anticipated poor bilateral spread of infused local anesthetic and subsequent inadequate pain relief. The

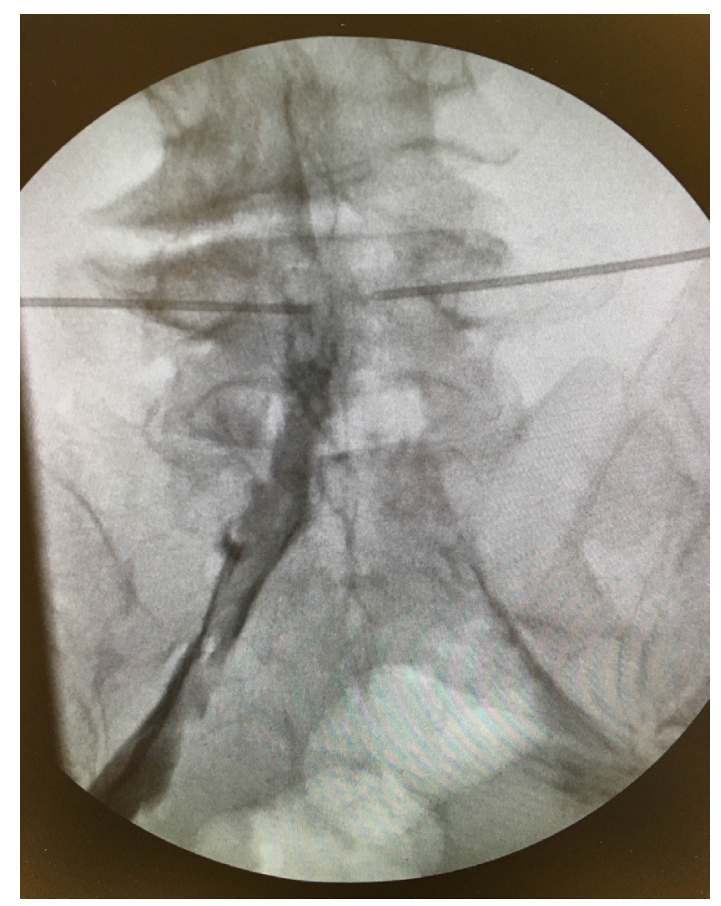

Figure 2 Anteroposterior fluoroscopic image of contrast injected through the second epidural catheter which was placed on the left side of the epidural space showing spread only to the left-sided nerve roots. 
patient tolerated the procedure well and was placed on dual patient controlled epidural analgesia (PCEA) pumps with each pump delivering a basal dose of $3 \mathrm{~mL}$ of bupivacaine $0.2 \%$ per hour with a demand dose of $3 \mathrm{~mL}$ every $30 \mathrm{~min}$ utes. She received excellent pain control and her reported numeric pain score for her lower extremity and sacral pain went from a 10 to a 3 . She was discharged to hospice and placed under the care of the palliative physician. On phone call follow-up, it was reported that she had no adverse effects associated with the epidural infusion and continued to have numeric pain scores in the 3 to 6 range. While in hospice she was continued on the same basal epidural infusion and with approximately five demand doses per day. The patient remained on the same topical and oral pain medication regimen, which included transdermal fentanyl and oral hydrocodone/acetaminophen. Unfortunately after 4 weeks, the patient passed away in her sleep with her husband at her bedside. Written informed consent was obtained from the patient's next of kin (husband) to publish this case report and any accompanying images.

\section{Discussion}

Although unilateral epidural analgesia is relatively rare, we must be aware of its possibility and adapt our decision making appropriately. This is an especially important consideration to achieve the best outcomes for patients with cancer-related pain. There have been many proposed mechanisms for the uneven distribution of local anesthetic causing unilateral analgesia including slow injection of small volumes of local anesthetic, acquired adhesions in the epidural space, misplacement of the catheter in the epidural space, and a dorsomedian septum in the lumbar epidural space. ${ }^{1,6-9}$ A substantial amount of disagreement still surrounds the hypothesis that a PMD often exists in the epidural space and that it clinically affects local anesthetic spread. Previous case reports have described unilateral analgesia associated with partitioning within the epidural space confirmed through imaging. ${ }^{7,10,11}$ The existence of PMD and its prevention of bilateral spread of contrast in the lumbar epidural space have been demonstrated using CT with contrast and epidurography. ${ }^{1,5,12,13}$ However, CT scans without contrast and histologic examinations have often failed to determine the composition of the PMD as no fibrous fold or septum was found in the epidural space. ${ }^{1-4,14}$ Alternatively, it has been proposed that the makeup of the structures in the epidural space is actually adipose tissue collections which are distorted by injectate and that any unilateral spread is most likely due to needle and catheter misplacement. ${ }^{2,3}$
Regardless of its composition, it is important to note that a PMD may not be the most important or only factor responsible for unilateral spread of local anesthetic.

The patient had intractable neoplasm-related sacral and pelvic pain secondary to compression and inflammation of nerves by tumors. Using dual epidural catheters with PCEA proved to be an effective and necessary technique in this patient to achieve bilateral analgesia. The decision to place bilateral epidural catheters was made only after ruling out malposition of the catheter and using a large volume test dose to confirm persistent unilateral analgesia. The advantages to the epidural analgesia technique include a proven history for the effectiveness for terminal cancer pain, the ability to confirm bilateral analgesia prior to the patient leaving the procedure room, and the ability to titrate dosing to maximize analgesia and minimize side effects. Disadvantages to placing two external catheters are that there is a potential increased risk of infection and complications associated with placement when compared to using a single catheter. Alternatives to this procedure could include a single intrathecal phenol ablation of spinal nerves or increasing oral opiate dosing. Another option could have been to remove the epidural catheter and replace it with a single intrathecal catheter for a direct infusion of analgesics into the CSF. Some limitations of these techniques include possible elimination of motor function and higher chance of intolerability of opiate side effects. Ultimately the patient received effective pain control for the remainder of her life. The dual epidural catheter technique for controlling terminal cancer pain should be considered in patients who receive insufficient analgesia from oral opioid and other palliative treatments.

\section{Acknowledgment}

The abstract of this paper was presented as a poster presentation at the North American Neuromodulation Society Annual Conference $2017 .{ }^{15}$

\section{Disclosure}

The authors report no conflicts of interest in this work.

\section{References}

1. Asato F, Goto F. Radiographic findings of unilateral epidural block. Anesth Analg. 1996;83(3):519-522.

2. Hogan Q. Epidural anatomy: new observations. Can J Anaesth 1998;45(5 Pt 2):R40-R48

3. Hogan Q. Epidural catheter tip position and distribution of injectate evaluated by computed tomography. Anesthesiology. 1999;90(4): 964-970.

4. Hogan Q. Lumbar epidural anatomy. A new look by cryomicrotome section. Anesthesiology. 1991;75(5):767-775. 
5. Savolaine ER, Pandya JB, Greenblatt SH, Conover SR. Anatomy of the human lumbar epidural space: new insights using CT-epidurography. Anesthesiology. 1988;68(2):217-220.

6. Srivastava U, Pilendran S, Dwivedi Y, Shukla V. Radiographic evidence of unilateral epidural anesthesia. J Anaesthesiol Clin Pharmacol. 2013;29(4):571-572.

7. Visser WA, Lee RA, Gielen MJ. Factors affecting the distribution of neural blockade by local anesthetics in epidural anesthesia and a comparison of lumbar versus thoracic epidural anesthesia. Anesth Analg. 2008;107(2): $708-721$.

8. Gallart L, Blanco D, Samso E, Vidal F. Clinical and radiologic evidence of the epidural plica mediana dorsalis. Anesth Analg. 1990;71(6):698-701.

9. Hermanides J, Hollmann MW, Stevens MF, Lirk P. Failed epidural: causes and management. Br J Anaesth. 2012;109(2):144-154.

10. Usubiaga J, Reis A, Usubiaga L. Case reports: epidural misplacement of catheters and mechanisms of unilateral blockade. Anesthesiology. 1970;32(2):158-161.
11. Boezaart AP. Computerized axial tomo-epidurographic documentation of unilateral epidural analgesia. Obstetric Anesthesia Digest. 1990;10(2):117.

12. Park WY. Factors influencing distribution of local anesthetics in the epidural space. Reg Anesth. 1988;13(2):49-57.

13. Yaeger MP, Bae EE, Parra MC, Barr PA, Bonham AK, Sites BD. Fluoroscopy-assisted epidural catheter placement: an exploratory analysis of 303 pre-operative epidurograms. Acta Anaesthesol Scand. 2016;60(4):513-519.

14. Harrison GR. Topographical anatomy of the lumbar epidural region: an in vivo study using computerized axial tomography. Br J Anaesth. 1999;83(2):229-234.

15. Padalia RB, Padalia DM, Reeves CJ, et al. Case report: bilateral tunneled epidural catheters to prevent unilateral analgesia for cancer-related pain. Poster presented at: North American Neuromodulation Society Annual Conference 2017; January 21, 2017.
Local and Regional Anesthesia

\section{Publish your work in this journal}

Local and Regional Anesthesia is an international, peer-reviewed, open access journal publishing on the development, pharmacology, delivery and targeting and clinical use of local and regional anesthetics and analgesics. The journal is included in PubMed, and welcomes submitted papers covering original research, basic science, clinical studies,

\section{Dovepress}

reviews and evaluations, guidelines, expert opinion and commentary, case reports and extended reports. The manuscript management system is completely online and includes a very quick and fair peer-review system, which is all easy to use. Visit http://www.dovepress.com/ testimonials.php to read real quotes from published authors. 